Revue internationale de l'économie sociale

Recma

Démocratiser l'économie, Hugues Sibille, Tarik Ghezali, Grasset. 2010, 138 pages

\title{
Danièle Demoustier
}

Numéro 320, avril 2011

URI : https://id.erudit.org/iderudit/1020916ar

DOI : https://doi.org/10.7202/1020916ar

Aller au sommaire du numéro

\section{Éditeur(s)}

Association Recma

\section{ISSN}

1626-1682 (imprimé)

2261-2599 (numérique)

Découvrir la revue

Citer ce compte rendu

Demoustier, D. (2011). Compte rendu de [Démocratiser l'économie, Hugues

Sibille, Tarik Ghezali, Grasset. 2010, 138 pages]. Revue internationale de

l'économie sociale, (320), 122-124. https://doi.org/10.7202/1020916ar d'utilisation que vous pouvez consulter en ligne.

https://apropos.erudit.org/fr/usagers/politique-dutilisation/ 
Enfin, l'ouvrage décrit les défis que doit relever l'action publique sur le financement agricole: la segmentation excessive des politiques de financement; les enjeux extra-agricoles; l'articulation d'actions aux niveaux local, régional, national, international; le manque d'information et de formation; la construction d'une position commune face au risque de conflit d'intérêts dans la gestion des services financiers; coordonner et rendre viables les services d'appui aux producteurs (ch.7). Les annexes méritent mieux que leur appellation: elles permettent de finaliser l'appropriation du guide à partir d'exemples très stimulants. Au-delà de son ambition opérationnelle, ce guide constitue également le meilleur argumentaire qui soit pour celles et ceux qui s'intéressent à la question du développement des agricultures familiales et souhaitent aller au-delà des critiques générales de l'agro-business ou des leçons plus ou moins simplistes tirées du seul bon sens. Sur le plan épistémologique, il témoigne de la fécondité de la démarche de recherche-actionformation à laquelle l'économie sociale est si attachée, fécondité qui s'exprime par l'articulation remarquable entre l'analyse distanciée et les propositions techniques et pratiques. Sur le fond, si vous pensez que 1) l'agriculture paysanne est indispensable pour nourrir le monde, 2) qu'elle ne peut se passer d'un soutien financier et 3) que les associations et les acteurs publics ont un rôle à jouer pour la soutenir, ce livre est pour vous. Si vous ne le pensez pas, ce livre va vous en convaincre.

Jean-François Draperi

\section{Démocratiser l'économie}

Hugues Sibille, Tarik Ghezali, Grasset. 2010, 138 pages.

Dans leur ouvrage consacré à la démocratisation del'économie, Hugues Sibille et Tarik Ghezali ${ }^{(1)}$ s'attaquent à une question récurrente dans notre histoire sociale: la démocratie économique,

(1) Respectivement: vice-président du Crédit coopératif, président de I'Avise et d'Ides-Esfin; délégué général du Mouves (www.mouves.org) et co-animateur du Labo de I'ESS. question familière aux praticiens et analystes de l'économie sociale et solidaire. Contrairement au discours des économistes qui réclament un retour de la régulation publique, ils affirment une conviction forte: la sortie de crise "ne sera pas keynésienne ». Ce ne sera pas une économie mixte, mais une économie plurielle qui nous permettra de sortir des dégâts opérés par les dérégulations. Dans ces nouvelles "régulations citoyennes et entrepreneuriales» (p. 10), les sociétés de personnes devraient jouer un rôle important pour contrebalancer le pouvoir des actionnaires et des managers qui a conduit à la financiarisation de l'économie. La perspective d'une plus forte valorisation de la coopération est séduisante, mais l'argumentation n'est pas dénuée d'ambiguïtés.

\section{La concurrence, seul moteur del'innovation?}

Si l'économie sociale apparait comme le socle historique de cette transformation, l'approche de l'économie solidaire est complètement absente (ce qui est particulièrement étonnant quand on se souvient que Hugues Sibille, alors délégué interministériel à l'économie sociale, a été un ardent militant du rapprochement entre économie sociale et économie solidaire à la fin des années 90). De ce fait, « la querelle des anciens et des modernes " opposerait une économie sociale historique (encore moderne ou dépassée car incapable de défendre des intérêts autres que ceux des couches incluses ou intégrées? Les deux appréciations se succèdent) et une société civile qui "se met en marche", notamment sous l'impulsion des "entrepreneurs sociaux innovants" (p. 53).

Le marché est en effet incontournable et il " oblige à se remettre en cause ». "Les entrepreneurs sont plus que d'autres amenés à se remettre en cause eux-mêmes" (p. 85); seuls les entrepreneurs sociaux sont attentifs aux nouveaux besoins sociaux. Nul ne doute que la concurrence est un puissant stimulant quand elle fonctionne réellement, mais elle est également sélective; en revanche, les auteurs semblent oublier un peu rapidement toute la capacité de mobilisation 
et d'innovation des multiples militants, bénévoles et usagers qui, dans les associations, tentent de transformer les nouveaux besoins en réponses appropriées à des groupes sociaux plus ou moins solvables.

\section{Ethique et autorégulation dans un marché non défini}

De ce fait, la morale est censée laisser la place à l'éthique, car l'individu est par nature un être social et ne semble pas avoir besoin de règles extérieures (notamment de statuts contraignants); l'autorégulation par des chartes, labels ou systèmes de notation extrafinancière devrait suffire à orienter son comportement vers l'intérêt général. La démocratie économique glisse alors rapidement dans deux directions: d'une part, vers l'information des "parties prenantes » et l'élaboration de nouveaux critères d'évaluation, sans réellement interroger le mode de gouvernance; d'autre part, vers l'accès aux droits économiques (travail, logement, consommation, crédit), accès supposé facilité par l'intégration au marché, sans aucune interrogation sur les limites de ce même marché à intégrer une partie croissante de la population (du marché du travail au marché du logement).

\section{De nouvelles alliances au sein d'un capitalisme patrimonial et non financier?}

Le dépassement de ces limites devrait être trouvé dans la constitution "de nouvelles alliances" (p. 97), de projets comme d'idées, vertueuses, avec des grandes entreprises soucieuses de responsabilité sociale alors que «le creuset de l'Etat républicain n'a pas généré une culture de partenariat " (p. 99). Parmi les exemples apportés (Darty, Danone, Mulliez...), certains nous interrogent: est-il plus démocratique de financer directement un soutien aux entreprises d'utilité sociale ou de payer des impôts qui servent à la redistribution et au financement des services publics, voire aux subventions à ces mêmes entreprises? Le sujet mérite débat.

Les auteurs se défendent de naïveté: "Nous ne sommes pas chez les Bisounours"; il ne s'agit pas de se substituer à l'Etat; ce n'est "pas le remède miracle aux insuffisances de la régulation publique " (p. 107), "il convient de privilégier le ET plutôt que le $O U$ ». Mais malheureusement, pour de nombreuses entreprises, le OU (financement direct) remplace le ET (financement par l'impôt) et, de ce fait, l'Etat n'a plus les moyens budgétaires suffisants.

Dans ce cadre, "l'intérêt général devient une affaire de copropriété " (p. 99), comme le territoire un "espace socio-économique en copropriété" " (p. 123). Le citoyen réduit au rôle de propriétaire et l'Etat à sa fonction de garant du droit de propriété, n'est-ce pas un retour au capitalisme patrimonial du XIX ${ }^{e}$ siècle?

Un certain malaise se dégage de la lecture de cet ouvrage: de quelle démocratie parlent les auteurs? Quelle est leur analyse du marché? de l'intervention de l'Etat? Quelle forme de coopération est encouragée: entre les membres et bénéficiaires? avec les grandes entreprises? Quelle professionnalisation des entreprises sociales?

Comme très souvent dans le secteur culturel, le mot "démocratie " est utilisé à la fois pour désigner la pratique (culturelle) et l'accessibilité (aux biens culturels), ce qui renvoie à des projets sensiblement différents. De même, que signifie "accepter le marché, mais pas le tout-marchand" au-delà de l'écho au débat "oni à l'économie de marché, non à la société de marché»" (p. 16), qui a montré que l'économie ne peut pas être réduite à un champ autonome dans la société?

L'intervention de l'Etat reste très floue: l'Etat garant (de quoi ?), régulateur (de la finance uniquement?), l'Etat qui ne disparaît pas (mais se transforme comment?). Il est en tout cas fortement concurrencé, dans le nouveau modèle économique sous-jacent, par les entreprises multinationales "responsables ": au nom de quoi? del'innovation? dela responsabilitésociale? de la rationalisation comptable et budgétaire?...

\section{Des perspectives contradictoires}

Enfin se pose la réelle question de la gouvernance: ne sagit-il pas, au nom de la performance, 
de la volonté de changement d'échelle..., de substituer au couple administrateurs élusprofessionnels techniciens une nouvelle génération de gestionnaires qui concentrent la plus grande partie des pouvoirs?

De toutes les questions qui émergent, il peut donc ressortir des perspectives très contradictoires: d'une part, l'individualisation de l'entreprise collective, autour de la figure de "l'entrepreneur ", et la marchandisation de l'action sociale (au sens large) dans le cadre d'une concurrence accrue pondérée par du mécénat d'entreprise; d'autre part, une plus grande «socialisation" de l'économie par la démocratisation et la coopération. C'était l'ambition affichée sur la couverture, ce n'est pas réellement la conclusion que l'on peut retenir du contenu de l'ouvrage. Certes, toute économie est complexe. Ce qui sortira de la crise sera probablement une imbrication nouvelle de ces deux logiques, mais glisser sans cesse de l'une à l'autre n'aide pas forcément le lecteur à percevoir plus précisément les grands enjeux politiques qui se profilent derrière les transformations en cours.

Danièle Demoustier 